\title{
Evaluation and monitoring of the air-sea interaction using a CBR-Agents approach
}

\author{
Javier Bajo ${ }^{1}$ and Juan M. Corchado ${ }^{2}$ \\ ${ }^{1}$ Universidad Pontificia de Salamanca \\ C/ Compañía 5 \\ 37002, Salamanca, Spain \\ jbajope@upsa.es \\ ${ }^{2}$ Departamento Informática y Automática \\ Universidad de Salamanca \\ Plaza de la Merced s/n \\ 37008, Salamanca, Spain \\ corchado@usal.es
}

\begin{abstract}
This paper presents a model constructed for the evaluation of the interaction of the atmosphere and the ocean. The work here presented focuses in the development of an agent based architecture that has been constructed for the evaluation of the interaction, between the atmosphere and the ocean waters, of several parameters. Such evaluation needs to be made continuously in a dynamic environment and therefore requires the use of autonomous models that evolve with the time. The proposed architecture incorporates CBR-agents whose aim is to monitor the evolution of the interaction of parameters and facilitate the creation of an explanation model. The system has been tested and this paper presents the results obtained.
\end{abstract}

\section{Introduction}

The agent paradigm is gaining relevance in the development of applications for flexible and dynamic environments, such as the web, personalized user interfaces, oceanography, control systems or robotic environments. Agents are often characterized by their capabilities such as autonomy, reactivity, pro-activeness, social ability, reasoning, learning, and mobility, among others. These capabilities can be modelled in different ways and with different tools [26], one of the possibilities is the use of CBR systems. This paper presents a CBR-agent based architecture that is the core of a distributed system developed for the analysis and evaluation of the interaction between ocean water masses and the atmosphere. The aim of this paper is to present a successful architecture that allows the construction of dynamic systems capable of growing in dimension and of adapting their knowledge to environmental changes. In this work we are mainly interested in the modelling of deliberative agents with CBR systems, as they can be used for implementing adaptive systems. Agents must be able to respond to events, which occur within their environment, take the initiative according to their goals, interact with other agents (even human), and to use past experiences to achieve current goals. Several architectures have been proposed 
for building deliberative agents, most of them based on the BDI model [21]. In this model, agents have mental attitudes of Beliefs, Desires and Intentions. In addition, they have the capacity to decide what to do and how to get it according to their attitudes. The beliefs represent its information state, what the agent knows about itself and its environment. The desires are its motivation state, what the agent is trying to achieve. And the intentions represent the agent's deliberative states. Intentions are sequences of actions; they can be identified as plans. These mental attitudes determine the agent's behaviour and are critical in attaining proper performance when the information about the problem is scarce [2, 15].

A BDI architecture has the advantage that it is intuitive and relatively simple to identify the process of decision-making and how to perform it. Furthermore, the notions of belief, desire and intention are easy to understand. On the other hand, its main drawback lies in finding a mechanism that permits its efficient implementation. There are several approaches to formalise and implement BDI agents, among them, dMARS [8], PRS [18], JACK [4], JAM [14], and AgentSpeak(L) [20]. One of the problems for an efficient implementation lies in the use of multi-modal logic for the formalisation and construction of such agents, because they have not been completely axiomatised and they are not computationally efficient. Rao and Georgeff [21] state that the problem lies in the great distance between the powerful logic for BDI systems and practical systems. Another problem is that this type of agent is not able to learn, a necessary requirement for them since they have to be constantly adding, modifying or eliminating beliefs, desires and intentions. It would be convenient to have a reasoning mechanism that would enable the agent to learn and adapt in real time, while the computer program is running, avoiding the need to recompile such an agent whenever the environment changes.

In order to overcome these issues, we propose the use of a case-based reasoning (CBR) system for the development of deliberative agents $[5,8]$. The proposed method facilitates the automation of their construction. Implementing agents in the form of CBR systems also facilitates learning and adaptation, and therefore a greater degree of autonomy than with a pure BDI architecture [13]. If the proper correspondence between the three mental attitudes of BDI agents and the information manipulated by a CBR system is established, an agent with beliefs, desires, intentions and a learning capacity will be obtained. Our approach to establish the relationship between agents and CBR systems differs from other proposals $[1,10,17,19,25]$, as we propose a direct mapping between the agent conceptualisation and its implementation, in the form of a CBR system.

The next section reviews the relationships that can be established between CBR and BDI concepts. Section three describes the environmental problem that motivates most of this research. Section four describes the multiagent based system developed paying special attention to the CBR-BDI agents constructed. Finally the conclusions and the some preliminary results are presented. 


\section{CBR-BDI Agents}

The purpose of case-based reasoning (CBR) is to solve new problems by adapting solutions that have been used to solve similar problems in the past. The deliberative agents, proposed in the framework of this investigation, use this concept to gain in autonomy and improve their problem solving capabilities. Figure 1 shows the activity diagram of a CBR-BDI agent for one of the possible actions, which is composed of a reasoning cycle that consists of four sequential phases: retrieve, reuse, revise and retain.

An additional activity, revision of the expert's knowledge, is required because the memory can change as new cases appear during this process. Each of these activities can be automated, which implies that the whole reasoning process can be automated to a certain extent [6]. According to this, agents implemented using CBR systems could reason autonomously and therefore adapt themselves to environmental changes.

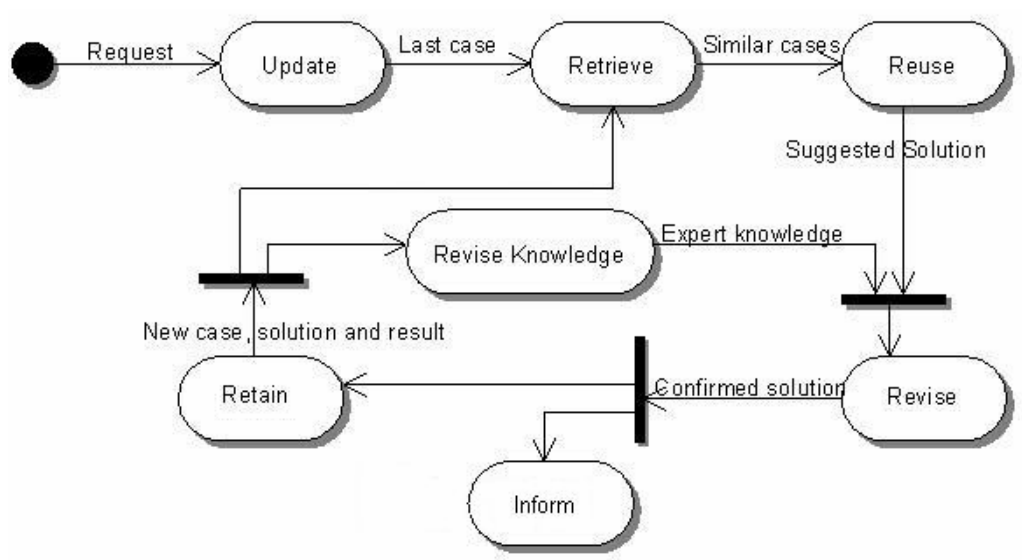

Fig. 1. Activity diagram describing a set of activities, including a CBR-BDI agent reasoning cycle.

The CBR system is completely integrated into the agents' architecture. The CBRBDI agents incorporate a "formalism" which is easy to implement, in which the reasoning process is based on the concept of intention. Intentions can be seen as cases, which have to be retrieved, reused, revised and retained. This makes this model unique in its conception and reasoning capacities. The structure of the CBR system has been designed around the concept of a case. A straight relationship between CBR systems and BDI agents can also be established if the problems are defined in the form of states and actions.

The relationship between CBR systems and BDI agents can be established implementing cases as beliefs, intentions and desires which led to the resolution of the problem. As described in [8], in a CBR-BDI agent, each state is considered as a belief; the objective to be reached may also be a belief. The intentions are plans of actions that the agent has to carry out in order to achieve its objectives [2], so an 
intention is an ordered set of actions; each change from state to state is made after carrying out an action (the agent remembers the action carried out in the past when it was in a specified state, and the subsequent result). A desire will be any of the final states reached in the past (if the agent has to deal with a situation, which is similar to a past one, it will try to achieve a similar result to the previously obtained result). In [8] can be seen a diagram with a representation of the relationship between BDI agents and CBR systems

\section{Quantification of the Ocean Interaction Budget}

An understanding of the natural sources and sinks of atmospheric carbon dioxide is necessary for predicting future atmospheric loading and its consequences for global climate. Present estimates of emissions and uptake do not balance, and although some have attributed the imbalance to a terrestrial sink, the magnitude of the oceanic sink remains undefined [22]. The vast amount of new data on atmospheric $\mathrm{CO}_{2}$ content and ancillary properties that have become available during the last decade and the development of mathematical models to interpret this data have lead to significant advances in our capacity to deal with such issues. However, a continuing major cause of uncertainty is the role played by photosynthesis in providing a sink for anthropogenic emissions [22]. The solution to these types of problems requires the use of dynamic systems, capable of incorporating new knowledge and facilitating the monitoring and estimation work carried out by oceanographers. The rapid increase in atmospheric $\mathrm{CO}_{2}$ resulting from atmospheric changes in the carbon cycle has stimulated a great deal of interest.

The need to quantify the carbon dioxide valence and the exchange rate between the oceanic water surface and the atmosphere has motivated us to develop a distributed system that incorporates CBR-BDI agents capable of estimating such values using accumulated knowledge and updated information. The CBR-BDI agents receive data from satellites, oceanographic databases, oceanic and commercial vessels. The information received is composed of satellite images of the ocean surface, wind direction and strength and other parameters such as water temperature, salinity and fluorescence.

The goal of our project is to construct a model that calculates the global budgets of $\mathrm{CO}_{2}$, a mean $\mathrm{CO}_{2}$ flux for the whole oceanographic basin. The oceans contain approximately 50 times more $\mathrm{CO}_{2}$ in dissolved forms than the atmosphere, while the land biosphere including the biota and soil carbon contains about 3 times as much carbon (in $\mathrm{CO}_{2}$ form) as the atmosphere [24]. The $\mathrm{CO}_{2}$ concentration in the atmosphere is governed primarily by the exchange of $\mathrm{CO}_{2}$ with these two dynamic reservoirs. Since the beginning of the industrial era, about 2000 billion tons of carbon have been released into the atmosphere as $\mathrm{CO}_{2}$ from various industrial sources including fossil fuel combustion and cement production. This amounts to about $35 \%$ of the pre-industrial level of approximately 590 billion tons as carbon. At present, atmospheric $\mathrm{CO}_{2}$ content is increasing at an annual rate of about 3 billion tons which corresponds to one half of the annual emission rate of approximately 6 billion tons 
from fossil fuel combustion. Whether the missing $\mathrm{CO}_{2}$ is mainly absorbed by the oceans or by the land and their ecosystems has been debated extensively over the past decade.

It is important, therefore, to fully understand the nature of the physical, chemical and biological processes which govern the oceanic sink/source conditions for atmospheric $\mathrm{CO}_{2}[16,24]$. Satellite-borne instruments provide high-precision, highresolution data on atmosphere, ocean boundary layer properties and ocean biogeochemical variables, daily, globally, and in the long term (Figure 2). All these new sources of information have changed our approach to oceanography and the data generated needs to be fully exploited. Wind stress, wave breaking and the damping of turbulence and ripples by surface slicks, all affect the air-sea exchange of $\mathrm{CO}_{2}$. These processes are closely linked to the "roughness" of the sea surface, which can be measured by satellite radars and microwave radiometers. Sea surface roughness consists of a hierarchy of smaller waves upon larger waves (photograph, left, and close-up, below). Different sensors give subtly different measurements of this roughness.
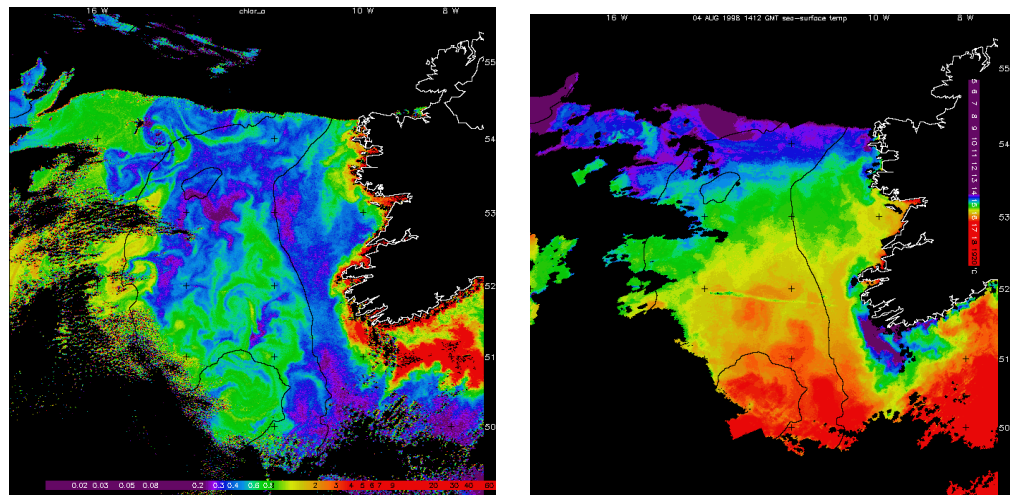

Fig. 2. Satellite colour pictures.

Our final aim is to model both the open ocean and shelf seas, and it is believed that by assimilating Earth Observation (EO) data into artificial intelligence models these problems may be solved. EO data (both for assimilation and for validation) are vital for the successful development of reliable models that can describe the complex physical and biogeochemical interactions involved in marine carbon cycling. Satellite information is vital for the construction of oceanographic models, and in this case, to produce estimates of air-sea fluxes of $\mathrm{CO}_{2}$ with much higher spatial and temporal resolution, using artificial intelligence models than can be achieved realistically by direct in situ sampling of upper ocean $\mathrm{CO}_{2}$. To handle all the potentially useful data to create daily models in a reasonable time and with a reasonable cost it is necessary the use of automated distributed systems capable of incorporate new knowledge. Our proposal is presented in the following section. 


\section{Multi Agent System}

Our scientific focus is on advancing the science of air-sea interactions and reducing the errors in the prediction of climate change. The primary goal is to quantify accurately the global air-sea fluxes of carbon dioxide. Over the past few years several models have been constructed and experiments carried out. Finally a distributed system has been constructed for solving the previously described problem. Gaia [27] has been initially used for the design of the distributed system, then AUML (Agentbased Unified Modelling Language) has been used for the low level design.

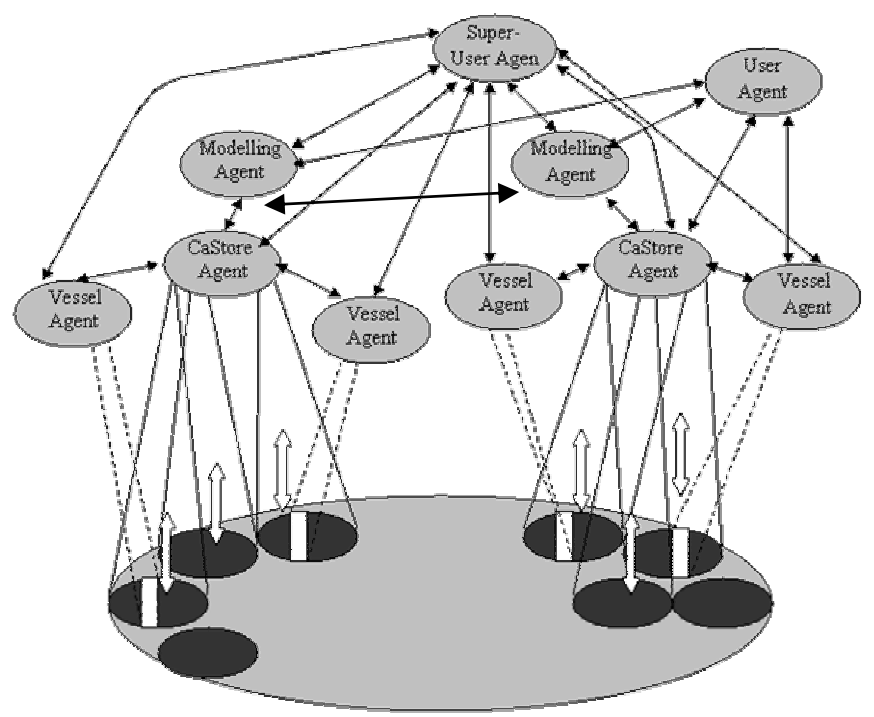

Fig. 3. Multiagente architecture.

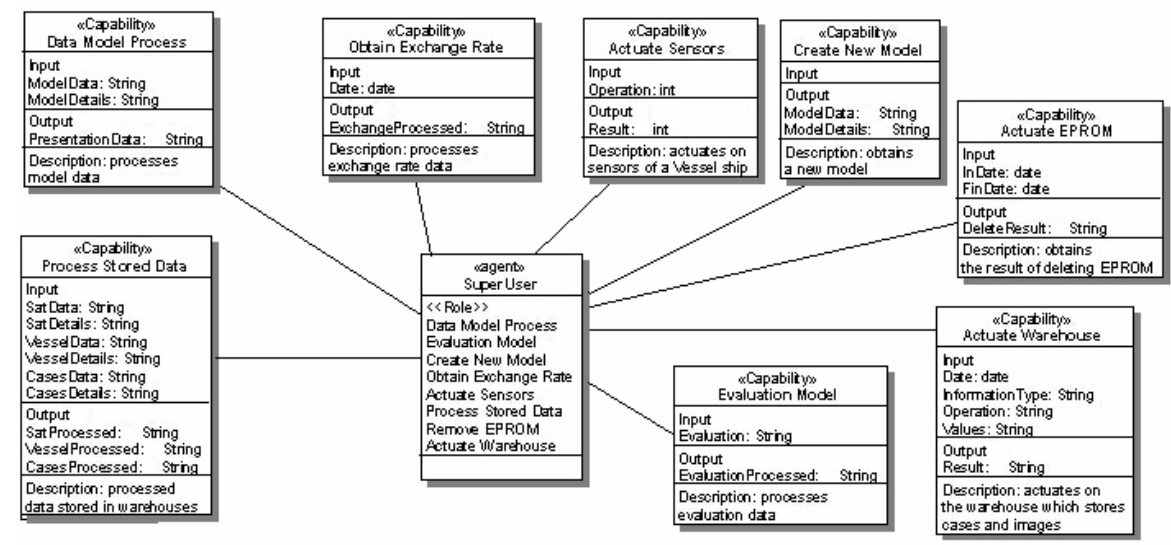

Fig. 4. Super User agent class diagram. 
This system incorporates several agents each of them in charge of a different task. Figure 3 presents an extension of the acquaintance model of a Gaia diagram and represents the multiagent architecture, its components and interactions. It incorporates reactive agents, in charge of repetitive tasks, and more complex deliberative CBRBDI agents in charge of monitoring, modelling and evaluating the interaction between the water surface and the atmosphere.

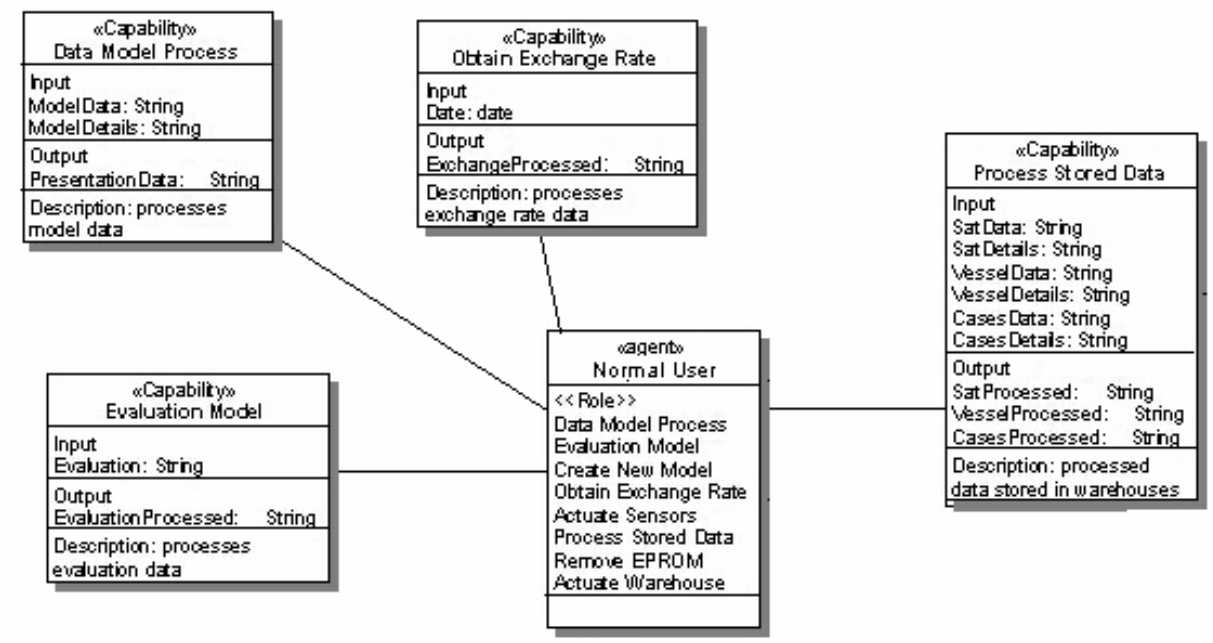

Fig. 5. User agent class diagram.

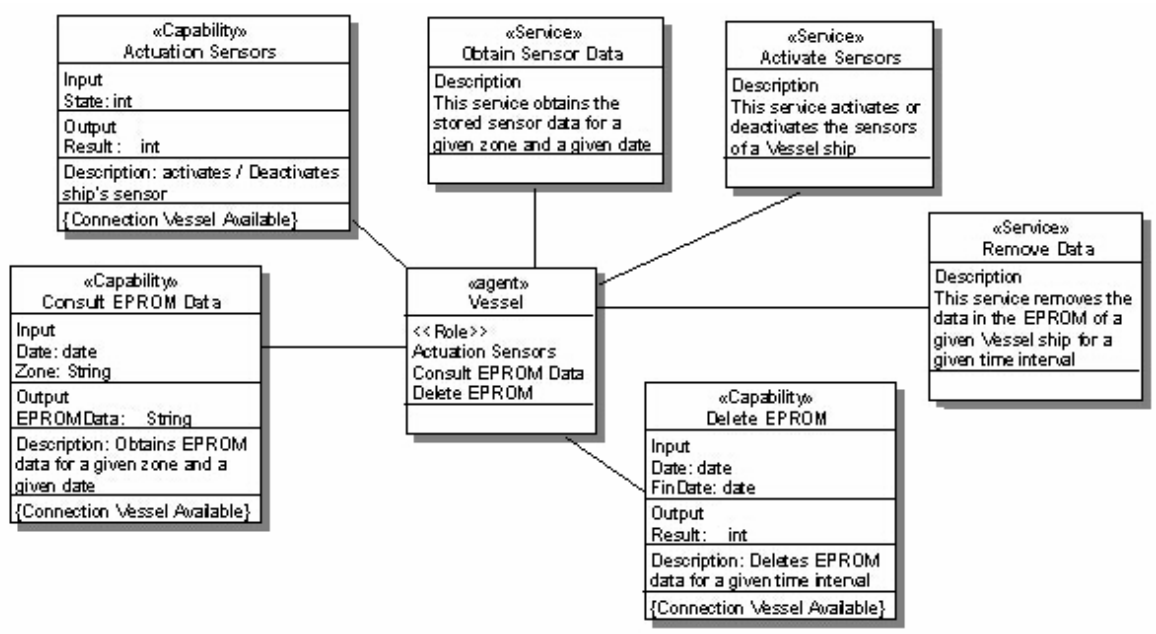

Fig. 6. Vessel agent class diagram.

The system includes a Super-user agent and several user agents, Modelling agents, CaStore agents and Vessel Agents. The User and Super-user are interface agents that facilitate the access to the system. Figure 4 and 5 show their AUML class diagrams. 
For example the Super-user agent facilitates the interaction between the super user and all the agents of the systems, as shown in Figure 4. These agents also facilitate the access to the data and to the constructed models. They also allow the users to follow and to modify the modelling process.

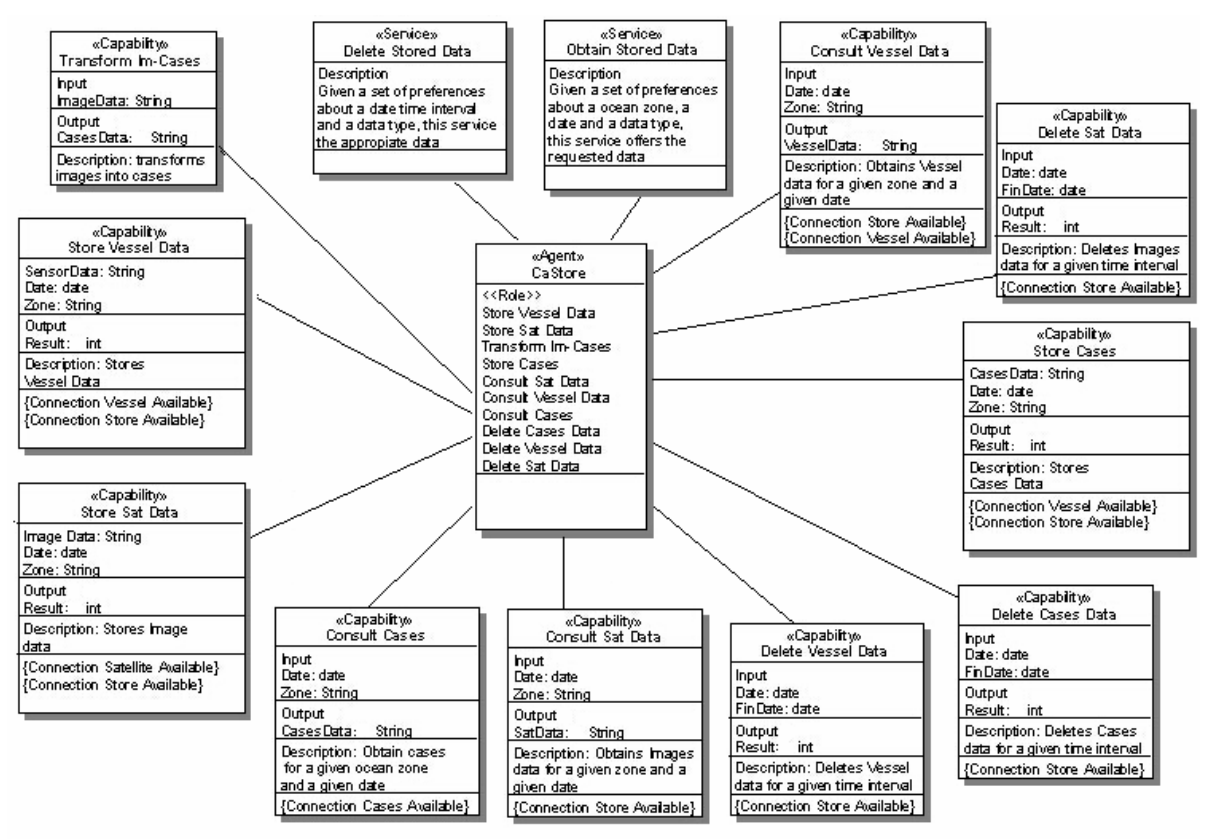

Fig. 7. CaStore agent class diagram.

The vessel agents are installed in the oceanographic and commercial ships that collaborate with the research project. They receive information from different sensors and store it. They can send their stored data to the CaStore agents on demand. The vessel agent may act on the boat sensors and EPRONS. They can also initiate an evaluation process of a given model. Models are always constructed by the Modelling agents. The CaSrore agents transform the satellite images and the data received form the vessel agents in cases. When new cases are constructed the Modelling agents are informed so the model in use may be improved. These agents also facilitate the user the access to the case store. The CaStore agents store historical and updated information incoming form the Vessel agents and from satellites. This data is sent to the Modelling agents, which have the goal of monitoring the ocean evolution and of evaluating the carbon dioxide interaction between the water surface and the atmosphere. The Modelling agents are CBR-BDI agents that use a CBR system to achieve their goals. Figure 8 shows the class diagram of these Modelling agents. The Modelling agents use CoHeL IBR system to achieve their goals [7]. The Cooperative Maximum Likelihood Hebbian Learning (CoHeL) method is a novel approach that features both selection, in which the aim is to visualize and extract information from 
complex, and highly dynamic data. The model proposed is a mixture of factor analysis and exploratory projection pursuit based on a family of cost functions proposed by Fyfe and Corchado [12] which maximizes the likelihood of identifying a specific distribution in the data while minimizing the effect of outliers. It employs cooperative lateral connections derived from the Rectified Gaussian Distribution [23] in order to enforce a more sparse representation in each weight vector. This method is used for the clustering of instances, and during the retrieval stage of the IBR cycle, the adaptation step is carried out using a radial basis function network. Finally, the system is updated continuously with data obtained from the CaStore agents. The CoHeL IBR system is described in [7].

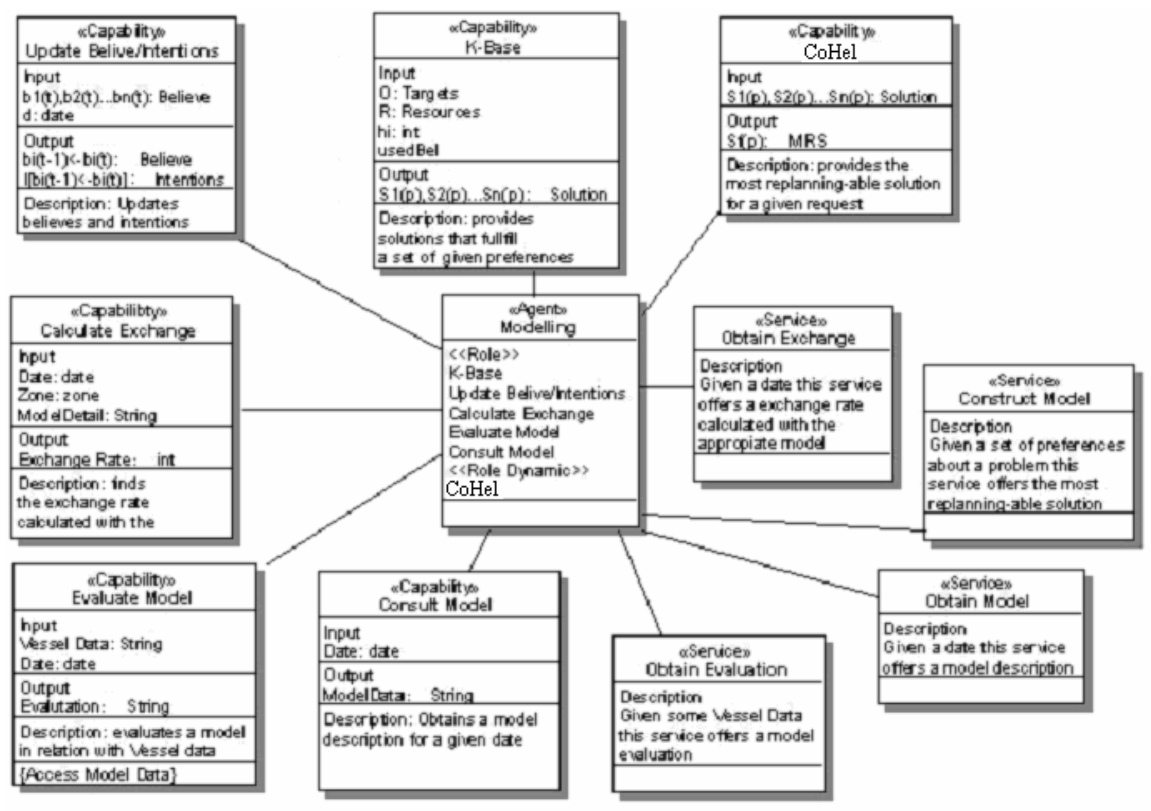

Fig. 8. Modelling agent class diagram.

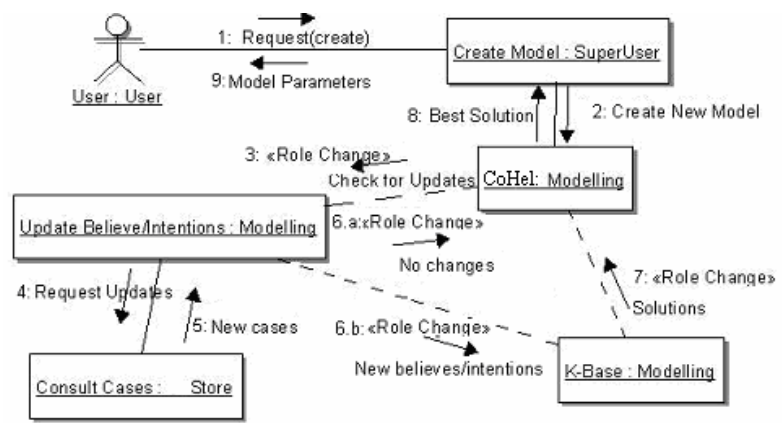

Fig. 9. Modelling agent interaction model, user request. 


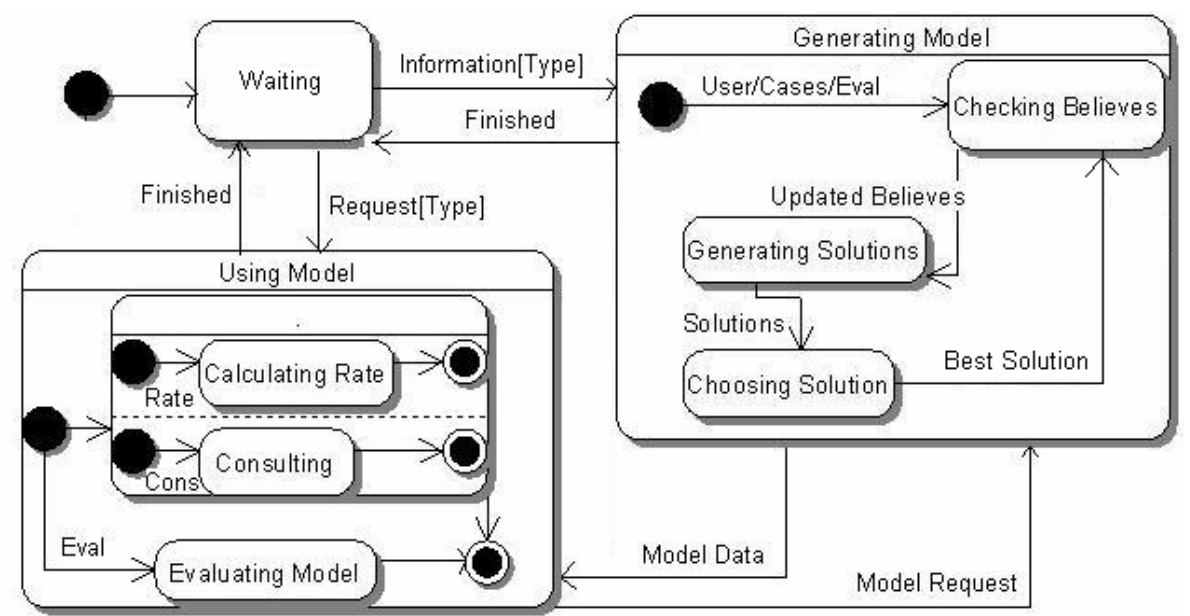

Fig. 10. State diagram for the Modelling agent.

Figure 9 shows one of the possible collaboration diagrams of the interaction model between the Modelling agent and the user of the system. This sequence of actions facilitates the construction of a model of the ocean surface - atmosphere interaction of a particular area of the ocean. Each Modelling agent is in charge of a particular oceanic area, for example the North Atlantic Ocean. The relationship between CoHel IBR systems and the BDI agent in which it is embedded can be established implementing instances such as beliefs, intentions and desires which lead to the resolution of the problem. When the Modelling agent starts to solve a new problem, with the intention of achieving a goal (identifying the carbon dioxide rate), it begins a new IBR reasoning cycle, which will help to obtain the solution. The retrieval, reuse and revision stages of the IBR system facilitate the construction of the agent plan. The agent's knowledge-base is the instance-base of the IBR system that stores the instances of past beliefs, desires and intentions. The agents work in dynamic environments and their knowledge-base has to be adapted and updated continuously by the retention stage of the IBR system. Figure 10 presents the state diagram for the Modelling agent. It shows how the agent is continually working and using the IBR life cycle for reasoning and achieving its goals. Figure 2 shows the activity diagram describing a CBR-BDI agent reasoning cycle, which can be applied to this agent too.

\section{System evaluation and conclusions}

The previously described system was tested in the North Atlantic Ocean during 2004. During this period of time the multiagent system has been tuned and updated and the first autonomous prototype started to work in may 2004. Although the system is not fully operational and the aim of the project is to construct a research prototype and not a commercial tool, the initial results have been very successful from the technical and scientific point of view. The construction of the distributed system has been relatively easy using previously developed CBR-BDI libraries [5, 6, 7, 8]. From the software 
engineering point of view AUML and Gaia [27] provide an adequate framework for the analysis and design of distributed agent based systems. The formalism defined in [13] facilitates the straight mapping between the agent definition and the CBR construction. The user can interact with the Modelling agent (via his/her user agent) following the interaction model described in Figure 10 and obtain information about the carbon dioxide exchange rate of a given area.

Table 1. Instance values.

\begin{tabular}{ll}
\hline Instance Field & Measurement \\
\hline DATE & Fecha \\
\hline LAT & Latitude \\
\hline LONG & Longitude \\
\hline SST & Temperature \\
\hline S & Salinity \\
\hline WS & Wind strength \\
\hline WD & Wind direction \\
\hline Fluo calibrated & fluorescence calibrated with chlorophyll \\
\hline SW pCO2 & surface partial pressure of $\mathrm{CO} 2$ \\
\hline
\end{tabular}

The Modelling Agents have their own interface and can also be accessed via the User or Super user agents. These agents handle beliefs, desires and intention from a conceptual point of view and cases from an implementation point of view. A case is composed of the attributes described in Table 1. Cases can be viewed, modified and deleted manually or automatically by the agent (during its revision stage). The agent plants (intentions) can be generated using different strategies since the agent integrates different algorithms.

Table 2. Million of Tones of $\mathrm{C} 02$ exchanged in the North Atlantic.

\begin{tabular}{l|l|l|l} 
& October 04 & November 04 & December 04 \\
\hline Multiagent System & -18 & 19 & $\mathbf{3 1}$ \\
\cline { 2 - 4 } Casix manual models & $\mathbf{- 2 0}$ & $\mathbf{2 5}$ & $\mathbf{4 0}$
\end{tabular}

The interaction between the system developers and oceanographers with the multiagent system has been continuous during the construction and pruning period, from December 2003 to September 2004. The system has been tested during the last three months of 2004 and the results have been very accurate. Table 2 presents the results obtained with the Multiagent systems and with mathematical Models [16] used be oceanographers to identify the amount of $\mathrm{CO} 2$ exchanged. The numerical values represent the million of Tonnes of carbon dioxide that have been absorbed (negative values) or generated (positive value) by the ocean during each of the three months.

The values proposed by the CBR-BDI agent are relatively similar to the ones obtained by the standard technique. In this case the case/instance base has been constructed with over 100,000 instances, and includes data since 2002. The multiagent system has automatically incorporated over 20,000 instances during these three months and eliminated $13 \%$ of the initial ones. While the CBR-BDI Modelling Agent generates results on a daily basis without any human intervention, the Casix manual modelling techniques require the work of one researcher processing data during al least four working days. Although the proposed system requires further 
improvements and more work the initial results are very promising. The CoHel IBR systems embedded within the Modelling agent has provided relatively accurate results in the North Atlantic Waters as well as in the Pacific Ocean [7]. The generated framework facilitates the incorporation of new agents using different modelling techniques and learning strategies so further experiment will allow to compare these initial results with the ones obtained by other techniques.

\section{References}

1. Bergmann, R. and W. Wilke (1996). On the role of abstraction in case-based reasoning. Lecture Notes in Artificial Intelligence, 1186, pp. 28-43. Springer Verlag.

2. Bratman M.E., Israel D., and Pollack M.E. (1988). Plans and resource-bounded practical reasoning. Computational Intelligence, 4. pages 349-355.

3. Bratman, M.E. (1987). Intentions, Plans and Practical Reason. Harvard University Press, Cambridge, M.A.

4. Busetta, P., Ronnquist, R., Hodgson, A., Lucas A. (1999). JACK Intelligent Agents Components for Intelligent Agents in Java. Technical report, Agent Oriented Software Pty. Ltd, Melbourne, Australia, 1998.

5. Corchado J. M. and Laza R. (2003). Constructing Deliberative Agents with Case-based Reasoning Technology, International Journal of Intelligent Systems. Vol 18, No. 12, December. pp.: 1227-1241

6. Corchado J. M. and Lees B. (2001). A Hybrid Case-based Model for Forecasting. Applied Artificial Intelligence. Vol 15, no. 2, pp.105-127.

7. Corchado J. M., Aiken J., Corchado E. Lefevre N. and Smyth T. (2004) Quantifying the Ocean's CO2 Budget with a CoHeL-IBR System. 7th European Conference on Casebased Reasoning, Lecture Notes in Computer Science, Lecture Notes in Artificial Intelligence 3155, Springer Verlag. pp. 533-546.

8. Corchado J. M., Pavón J., Corchado E. and Castillo L. F. (2005) Development of CBRBDI Agents: A Tourist Guide Application. 7th European Conference on Case-based Reasoning 2004. Lecture Notes in Artificial Intelligence 3155, Springer Verlag. pp. 547559 .

9. D'Iverno, M., Kinny, D., Luck, M., and Wooldridge, M. (1997). A Formal Specification of dMARS. In: Intelligent Agents IV, Agent Theories, Architectures, and Languages, 4th International Workshop, ATAL '97, Providence, Rhode Island, USA, July 24-26, 1997, Proceedings. Lecture Notes in Computer Science 1365, Springer Verlag, pp. 155-176.

10. Feret M. P. and Glasgow J. I. (1994). Explanation-Aided Diagnosis for Complex Devices, Proceedings of the 12th National Conference an Artificial Intelligence, (AAAI-94), Seattle, USA, August 94.

11. Freedman J. and Tukey J. (1974) A Projection Pursuit Algorithm for Exploratory Data Analysis. IEEE Transaction on Computers, (23): 881-890, 1974.

12. Fyfe C. and Corchado E. S. (2002) Maximum Likelihood Hebbian Rules. European Symposium on Artificial Neural Networks. 2002.

13. Glez-Bedia M., Corchado J. M., Corchado E. S. and Fyfe C. (2002) Analytical Model for Constructing Deliberative Agents, Engineering Intelligent Systems, Vol 3: pp. 173-185.

14. Huber, M. (1999). A BDI-Theoretic Mobile Agent Architecture. AGENTS '99. Proceedings of the Third Annual Conference on Autonomous Agents, May 1-5, 1999, Seattle, WA, USA. ACM, pp. 236-243. 
15. Kinny, D. and Georgeff, M. (1991). Commitment and effectiveness of situated agents. In: Proceedings of the Twelfth International Joint Conference on Artificial Intelligence (IJCAI'91), Sydney, Australia, pp. 82-88.

16. Lefevre N., Aiken J., Rutllant J., Daneri G., Lavender S. and Smyth T. (2002) Observations of pCO2 in the coastal upwelling off Chile: Sapatial and temporal extrapolation using satellite data. Journal of Geophysical research. Vol. 107, no. 0

17. Martín F. J., Plaza E., Arcos J.L. (1999). Knowledge and experience reuse through communications among competent (peer) agents. International Journal of Software Engineering and Knowledge Engineering, Vol. 9, No. 3, 319-341.

18. Myers, K. (1996). A Procedural Knowledge Approach to Task-Level Control. Proceedings of the Third International Conference on Artificial Intelligence Planning Systems,, pp. 158-165.

19. Olivia C., Chang C. F., Enguix C.F. and Ghose A.K. (1999). Case-Based BDI Agents: An Effective Approach for Intelligent Search on the World Wide Web, AAAI Spring Symposium on Intelligent Agents, 22-24 March 1999, Stanford University, USA.

20. Rao, A. S. (1996). AgentSpeak(L): BDI Agents speak out in a logical computable language. Agents Breaking Away, 7th European Workshop on Modelling Autonomous Agents in a Multi-Agent World, Eindhoven, The Netherlands, January 22-25, 1996, Proceedings. Lecture Notes in Computer Science 1038, Springer Verlag, pp. 42-55.

21. Rao, A. S. and Georgeff, M. P. (1995). BDI Agents: From Theory to Practice. First International Conference on Multi-Agent Systems (ICMAS-95). San Franciso, USA.

22. Sarmiento J. L. and Dender M. (1994) Carbon biogeochemistry and climate change. Photosynthesis Research, Vol. 39, 209-234.

23. Seung H.S., Socci N.D. and Lee D. (1998) The Rectified Gaussian Distribution, Advances in Neural Information Processing Systems, 10.

24. Takahashi T., Olafsson J., Goddard J. G., Chipman D. W. and Sutherland S. C. (1993) Seasonal Variation of $\mathrm{CO} 2$ and nutrients in the High-latitude surface oceans: a comparative study. Global biochemical Cycles. Vol. 7, no. 4. pp 843-878.

25. Wendler J. and Lenz M. (1998). CBR for Dynamic Situation Assessment in an AgentOriented Setting. Proc. AAAI-98 Workshop on CBR Integrations. Madison (USA) 1998.

26. Wooldridge, M. and Jennings, N. R. (1995) Agent Theories, Architectures, and Languages: a Survey. In: Wooldridge and Jennings, editors, Intelligent Agents, SpringerVerlag, pp. 1-22.

27. Wooldridge, M. and Jennings, N. R. and Kinny, D. (2000) The Gaia Methodology for Agent-Oriented Analysis and Design. Journal of Autonomous Agents and Multi-Agent Systems, 3 (3). pp. 285-312. 